\title{
A concept map of death-related anxieties in patients with advanced cancer
}

\author{
Sigrun Vehling, Carmine Malfitano, Joanna Shnall, Sarah Watt, \\ Tania Panday, Aubrey Chiu, Anne Rydall, Camilla Zimmermann, \\ Sarah Hales, Gary Rodin, and Christopher Lo
}

Version Post-print/accepted manuscript

Citation Vehling S, Malfitano C, Shnall J, Watt S, Panday T, Chiu A, Rydall A, (published version) Zimmermann C, Hales S, Rodin G, Lo C.A concept map of deathrelated anxieties in patients with advanced cancer.BMJ Supportive \& Palliative Care. 2017 Dec;7(4):427-434.

doi:10.1136/bmjspcare-2016-001287

Copyright information (C) Article author(s) (or their employer(s) unless otherwise stated in the text of the article) 2017. All rights reserved. No commercial use is permitted unless otherwise expressly granted.

How to cite TSpace items

Always cite the published version, so the author(s) will receive recognition through services that track citation counts, e.g. Scopus. If you need to cite the page number of the author manuscript from TSpace because you cannot access the published version, then cite the TSpace version in addition to the published version using the permanent URI (handle) found on the record page.

This article was made openly accessible by $U$ of $T$ Faculty.

Please tell us how this access benefits you. Your story matters. 
Full citation and link to final published version:

Vehling S, Malfitano C, Shnall J, Watt S, Panday T, Chiu A, Rydall A, Zimmermann C, Hales S, Rodin G, Lo C. A concept map of death-related anxieties in patients with advanced cancer. BMJ Supportive \& Palliative Care. 2017 Dec; 7(4): 427-434. doi:10.1136/bmjspcare-2016-001287 [Epub 2017 Aug 2]. 
Article type: Research paper

Title: A concept map of death-related anxieties in patients with advanced cancer

Running head: Concept map of death-related anxieties

Authors: $\quad$ Vehling, S. $\quad$, Malfitano, C. ${ }^{1}$, Shnall, J. ${ }^{1}$, Watt, S. ${ }^{1}$, Panday, T. ${ }^{1}$, Chiu, A. ${ }^{1}$, Rydall,

A. ${ }^{1}$, Zimmermann, C. ${ }^{1,2,3}$, Hales, S. ${ }^{1,2}$, Rodin, G. ${ }^{1,2}$, Lo, C. ${ }^{1,2,4}$

Affiliations: $\quad{ }^{1}$ Department of Supportive Care, Princess Margaret Cancer Centre, University

Health Network, Toronto, ON, Canada

${ }^{2}$ Department of Psychiatry, University of Toronto, Toronto, ON, Canada

${ }^{3}$ Department of Medicine, University of Toronto, Toronto, ON, Canada

${ }^{4}$ Department of Psychology, University of Guelph-Humber, Toronto, ON, Canada

Word count (excluding tables, figure captions, and references): 3444

Number of tables: 1

Number of figures: 3

\section{Corresponding author:}

Sigrun Vehling

c/o Department of Supportive Care

Princess Margaret Cancer Centre, University Health Network

610 University Avenue, 16-724

Toronto, ON, M5G 2M9, Canada

Phone: $+49(0) 40741056805$

E-Mail: s.vehling@uke.de 


\begin{abstract}
Objectives: Fear of death and dying is common in patients with advanced cancer, but can be difficult to address in clinical conversations. We aimed to show that the experience of death anxiety may be deconstructed into a network of specific concerns, and provide a map of their interconnections to aid clinical exploration.
\end{abstract}

Methods: We studied a sample of 382 patients with advanced cancer recruited from outpatient clinics at the Princess Margaret Cancer Centre, Toronto, Canada. Patients completed the 15-item Death and Dying Distress Scale (DADDS). We used item ratings to estimate a regularized partial correlation network of death and dying-related concerns. We calculated node closeness-centrality, clustering, and global network characteristics.

Results: Death-related anxieties were highly frequent, each associated with at least moderate distress in $22 \%$ to $55 \%$ of patients. Distress about "Running out of time" was a central concern in the network. The network was organized into two areas: one of more practical fears concerning the process of dying, and another of more psychosocial or existential concerns including relational problems, uncertainty about the future and missed opportunities. Both areas were yet closely connected via important bridges, e.g., fear of suffering and a prolonged death was linked to fear of burdening others.

Conclusions: Individuals with advanced cancer may have multiple interconnected death-related concerns, forming patterns that vary with individual priorities and fears. The bridging links between more practical and more psychosocial concerns emphasize that the alleviation of death anxiety may require interventions that combine symptom management, advance care planning, and psychological treatment approaches.

Key words: Death anxiety, death distress, advanced cancer, psycho-oncology, network analysis, end of life 


\section{Introduction}

Extended survival and the growing emphasis on palliative care have highlighted the importance of conversations about death and dying in advanced incurable cancer. Death-related concerns and fears are inherent in discussions about advance care planning and preferences for prognostic disclosure, and may also trigger the search for meaning in face of impending mortality [1,2]. For patients nearing the end of life, the possibility to discuss death and dying in a safe place can facilitate adaptation and prevent severe psychological and existential distress [3]. However, the evaluation of clinical conversations and supportive interventions that aim to alleviate death anxiety are limited by our understanding of this concept [4].

In advanced cancer, the understanding of death anxiety has been limited by the view that talking about this difficult topic may be demoralizing for patients. While this perspective has been related to modern medicine's emphasis on cure [5], it is also evident in traditional theories of death anxiety. These have often assumed that death awareness is an intolerable human experience and that selfdistancing and denial of death are necessary to maintain psychological health $[6,7]$. However, many patients with advanced cancer prefer open discussions about death-related concerns and timely preparation for the end of life $[8,9]$. This observation suggests that exploration of patients' deathrelated concerns and fears is essential to clinical interventions in this population [10-13]. Empirical evidence that may guide such exploration remains scarce [1].

Although death anxiety has been viewed as an overarching construct, it may also be deconstructed into particular fears and concerns about death and dying. Accordingly, the Death and Dying Distress Scale (DADDS [14]) captures concerns about the loss of time and missed opportunities in life, uncertainty about the future, fears about the process of dying, and the impact of one's death on others. Although factor analyses of the DADDS have found a single-factor structure [14, 15], qualitative studies and conceptual reviews point to a complex system of interacting thoughts, fears, and worries associated with death and dying in advanced cancer [4, 16-18]. Also, while studies have suggested a 
distinction of the fear of dying from the fear of death itself, the association between these domains is not well understood $[17,19,20]$.

The present study is focused on particular death-related fears and concerns and their links among patients with advanced cancer. We investigated the network structure of death-related concerns by a detailed analysis of the connections between them. This network approach thus "unpacks" the relatively abstract construct of death anxiety by closely examining the interrelation of its constituent elements. Related existential distress constructs such as demoralization, loss of dignity, and end-oflife preparation have been studied in advanced life-threatening disease [21-23]. However, relationships between existential concerns on the item level have rarely been examined [24].

We analyzed the network structure of death-related anxieties in patients with advanced cancer and we aimed to: (a) identify the presence of central concerns that connect together a large number of other concerns; (b) characterize the overall configuration of the network by describing the areas formed by closely interconnected concerns; and (c) interpret the role of bridge concerns that link those areas. The product of this analysis is a map of death-related concerns and their interconnections, which may be helpful in guiding the exploration and treatment of death anxiety in patients with advanced cancer near the end of life.

\section{Methods}

\section{Participants and procedures}

Patients with advanced cancer were recruited from outpatient oncology clinics at the Princess Margaret Cancer Centre, University Health Network (UHN) in Toronto, Canada. Advanced cancer was defined by diagnosis of a stage IV solid tumor or stage III lung or ovarian tumor, or any stage pancreatic cancer (due to aggressiveness of this disease), all with an expected survival of 12-18 months. Patients were invited to participate in a randomized controlled trial of a psychotherapeutic 
intervention called CALM (Managing Cancer And Living Meaningfully) to alleviate distress (for study protocol, see [25]). After providing written informed consent, participants completed baseline self-reported measures, including the assessment of death-related concerns or anxieties, which are the focus of the present study. Exclusion criteria were age younger than 18 years, severe cognitive or physical impairment, language barrier, and current receipt of other psychosocial interventions from the Centre's Department of Supportive Care. Patients provided written informed consent and completed baseline measures before randomization. Three hundred and eighty-two out of 2601 eligible patients with advanced cancer (15\%) completed baseline death anxiety questionnaires. This study was approved by the UHN Research Ethics Board.

\section{Measures}

Demographic data were collected by a standardized questionnaire. Disease-related characteristics were obtained from medical charts. We used the 15-item Death and Dying Distress Scale (DADDS) $[14,15]$ to assess death anxiety. The scale was specifically developed to capture concerns related to death and dying in patients with advanced cancer. It encompasses psychosocial and existential concerns about missed opportunities and the loss of time, past regrets and uncertainty in the future, and the impact or burden of death on others. Further, it assesses more practical concerns about the process of dying, including fears about dying alone or with suffering, or worries about its timing. Items are answered on a 6-point Likert scale from 0 (no distress) to 5 (extreme distress). Total scores may range from 0 to 75 and scores $\geq 45$ refer to the presence of at least moderate death anxiety. By inquiring about the specific death-related concerns of most relevance to patients with advanced cancer, the DADDS is distinct from death anxiety scales developed for use in non-advanced cancer populations for whom mortality is not imminent [15]. It focuses on the assessment of disturbing thoughts that require alleviation, and does not assess reaction to symbolic reminders of death nor death attitudes, for which the direction of desired clinical change is less clear (e.g., belief in an 
afterlife, death acceptance). This reflects a perspective on death anxiety as becoming more focused on the fear of death among patients with advanced disease, given the imminence of mortality and the many foreseeable challenges associated with dying [6].

\section{Statistical analysis}

We used R version 3.3.0 [26] and package qgraph, version 1.3.3 [27]. Descriptive statistics including means, standard deviations, and frequencies were calculated for the total scale as well as individual DADDS items. Pearson correlations were calculated between each pair of items given non-skewed distributions. Missing values occurred in only 2 of 5685 responses and these were mean-imputed.

Box 1: Introduction to the network approach We applied a network analysis perspective to death anxiety [28]. A network analysis examines the intercorrelations between concerns or symptoms in a graphical form. In a network graph, stronger links due to higher correlations between concerns are indicated by shorter and thicker lines (called "edges") connecting them. Network structures thus graphically illustrate which concerns tend to co-occur. They focus on the shape and interconnectivity of symptoms - unlike factor analysis in which is closely related symptoms are grouped to define discrete syndromes. Network structures are suggestive of how effective alleviation of a particular symptom (called a "node") may require addressing closely related symptoms in the network. In that regard, bridge symptoms that link otherwise separate areas of a network are an important feature and can indicate how intervention effects may transfer across network domains. 
We conducted a network analysis (see Box 1 for an introduction) of the 15 DADDS items to estimate and visualize the interrelationships between these concerns. A network analysis begins by calculating the partial correlations between each pair of items, that is, pairwise associations that have been adjusted for the effects of the other items in the network. This partial correlation matrix is visualized in a graph where items are displayed as nodes in the network and the partial correlations become the edges connecting these nodes. Thicker edges indicate higher partial correlations between nodes.

Highly correlated nodes are placed closer together through the force-directed Fruchterman-Reingold algorithm implemented in qgraph [27]. The sensitivity of the partial correlation matrix was optimized using a graphical lasso (least absolute shrinkage and selection operator) regularization technique. The glasso shrinks very small partial correlations to zero to maintain a sparse partial correlation matrix. The tuning parameter $\alpha$ returning the optimal degree of shrinkage is determined through an algorithm that minimizes the extended Bayesian information Criterion (EBIC). The hypertuning parameter $\gamma$, defining the degree of simplicity preferred by the EBIC, was set to the default medium value of 0.25 .

We further calculated descriptive indices to characterize the network's node-specific and global properties. At the local level, a node's closeness-centrality describes its total distance to all other nodes in the network. Higher values indicate greater centrality of the node within the network. A node's closeness is calculated by the inverse sum of its distances to all other nodes. The clustering coefficient describes the number of nonzero connections relative to the maximum number of possible connections between the neighbors of each node. Clustering can be interpreted as a measure of a node's redundancy. Values may range from 0 (no clustering) to 1 (high clustering). Barrat's coefficient was used to adjust for both edge width and number when calculating clustering coefficients. At the global level, the average shortest path length (ASPL) measures the average shortest number of edges between each pair of nodes. For example, a network with ASPL=2 means that on average, the shortest link between each pair of nodes is two edges. If ASPL=1, the network is highly concentrated and, on average, nodes are linked by only one edge. We further calculated Opsahl's weighted version of the ASPL, which adjusts for edge width. The transitivity of the network 
is a measure of its global clustering. It describes the average probability that two nodes, which are linked to the same neighbor, are neighbors themselves. Values may range between 0 and 1 . The smallworldness index (SWI) relates a network's transitivity to its unweighted ASPL. A smallworld network is characterized by high transitivity (i.e., high clustering) and low ASPL. Smallworldness is indicated by an $\mathrm{SWI} \geq 3$.

\section{Results}

Patient characteristics and frequency of death anxiety

382 patients completed baseline death anxiety questionnaires. Table 1 presents demographic and disease-related characteristics of this sample. Figure 1 shows the frequency of patients reporting each death-related concern (cut-off $\geq 3$ indicating at least moderate distress). The most frequent concerns were: the impact of one's death on loved ones $(65.4 \%)$; that one's death and dying may be prolonged or drawn out (55.3\%); and that it may happen with a lot of pain and suffering (54.8\%). Of note, even the least frequent concern (fear of dying alone) was reported by almost a quarter of participants. Further descriptive statistics of concerns are reported in the online supplement, Table 2. Patients reported having an average of $6.5(\mathrm{SD}=4.7)$ out of 15 possible death-related concerns. The mean total death anxiety score was $31.6(\mathrm{SD}=18.0)$. Moderate death anxiety was present in $27.4 \%$ of the patients (cut-off $\geq 45$ ).

Table 1: Demographic and disease related sample characteristics $(\mathrm{N}=382)$.

\begin{tabular}{lcc}
\hline Variable & N & \% \\
\hline Age, mean (SD) & $58.7(11.4)$ & \\
Gender & & \\
$\quad$ Female & 228 & 59.7 \\
$\quad$ Male & 154 & 40.3 \\
Having children & 269 & 70.4 \\
$\quad$ Having children under 18 & 64 & 16.8 \\
Cohabitation & 258 & 70.5 \\
\hline
\end{tabular}




\begin{tabular}{lrr}
\hline Education & & \\
Less than high school & 5 & 1.3 \\
High school & 69 & 18.1 \\
College/Trade & 90 & 23.6 \\
University & 202 & 52.9 \\
Tumor diagnosis & & \\
Gastrointestinal & 100 & 26.2 \\
Gynecological & 97 & 25.4 \\
Genitourinary & 61 & 15.9 \\
Lung & 49 & 12.8 \\
Breast & 33 & 8.6 \\
Other & 42 & 11.0 \\
No. of physical symptoms, mean (SD) & a & $12.31(4.9)$ \\
\hline
\end{tabular}

${ }^{a}$ Possible range: 0-28, according to the Memorial Symptom Assessment Scale [29]

Please insert Figure 1 about here

\section{Network of death-related anxieties}

Figure 2 presents the regularized partial correlation network of death-related concerns. The network has at least four major features in terms of structural configuration, concern areas, and bridge connections that facilitate "traveling" across areas in the map. The first important structural feature is that "Running out of time" (item 7) is located at the center of the network. Centrality is a different indication of item importance than frequency. A central item is one that retains the most relationship to all other concerns, after adjusting for all associations. Although it was not the most frequent concern, this node had the highest centrality value meaning that it was the most conceptually connected concern in the network (see Figure 3). The remaining concerns formed a circular chain around the "Running out of time" node. This circular arrangement is a reflection of the distribution of centrality values shown in Figure 3, i.e., less central concerns are placed more distant from the center. 
Please insert Figure 2 about here

Please insert Figure 3 about here

The second feature in Figure 2 describes a possible organization of the total network into two halves or areas, which coincides with differences in item content. The left area included all five items inquiring about practical fears about the dying process (grey-colored items 11 to 15). The right area included all, in a narrower sense, psychosocial and existential concerns related to the anticipation of death (items 1 to 10). The third feature of the network then showed how these concern areas of the map were connected via important bridges. Three main bridges (network edges) connected the left more "practical" and right more "psychosocial/existential" area (item 13 to 2, 15 to 7, and 12 to 8). The nodes at the ends of these edges can be viewed as key or bridge nodes that are travelled through to move across the network areas. This means that, for example, starting from fear of a prolonged death (item 12), exploration toward the right psychosocial area is possible via the closely related concern of being a burden to others (item 8).

A fourth noteworthy feature of the network is evident from examining the outer ring of death-related concerns surrounding "Running out of time". It describes a further possible organization of the items of this ring into four areas or quadrants. Starting at the upper right, the first quadrant was composed of items associated with past regrets and unfinished business, i.e. items $2,1,3$, and 6 . The second quadrant was composed of items relating to the future and uncertainties near the end of life, i.e. items 5,4 , and 10 . The third quadrant referred to items describing a prolonged and painful death and 
dependency needs in this circumstance, i.e. items $9,8,12$, and 14 . The fourth quadrant included items describing aspects of an unprepared death, specifically one that will happen soon, unexpectedly, and in the absence of family and friends, i.e. items 15, 11, and 13. As shown in Figure 3, clustering coefficients were high for concerns 3 and 14, indicating that these were most redundant in the network given closely interconnected neighbors.

Global network characteristics indicated a "mediumworld" structure of death anxiety. This means that concerns were neither extremely clustered (i.e., redundant) nor extremely distant (i.e., conceptually unrelated). The total number of nonzero edges in the network was 64 (61\% of possible edges). The average shortest path length between two nodes in the network was 1.4. That is, approximately half of node pairs were directly connected via one edge, while the other half were connected via two edges. The average weighted shortest path length was 12.5 , which was approximately equal to the weighted distance between concerns 7 and 12. The transitivity (global clustering coefficient) was 0.60 , indicating that $60 \%$ of nodes that shared a neighbor were themselves neighbors. The smallworldness index was 1.05; the network did hence not show smallworld properties.

\section{Discussion}

Although death anxiety has been viewed as an overarching concept, it may be deconstructed into a network of more specific fears or concerns, which in turn may be complexly interrelated. Using a network approach, this study graphically depicted the interconnections between 15 death-related concerns in a large sample of patients with advanced cancer. By visualizing the associations between concerns, the network may serve as a guide for clinicians and researchers to explore the structure of death-related anxieties in patients with advanced disease [30]. The interconnections between nodes suggest potential pathways along which the discussion of death-related fears may proceed. Although such conversations may begin anywhere, the centrality of the concern that time is running out suggests that starting the conversation here might allow for maximum flexibility in how the 
discussion unfolds. The "mediumworldness" of the network and similar centrality of remaining concerns indicate that each issue is non-redundant and worthy of exploration.

Although physical and practical concerns about dying clustered somewhat separately from psychosocial concerns, these network areas were also connected by clear pathways or bridges, suggesting that treatments to alleviate death anxiety may require addressing physical and psychosocial issues jointly. Although psychotherapeutic treatments [31] and palliative care interventions [32] have been developed to alleviate suffering and promote preparation for the end of life, their integration is less common. It is possible that integrated approaches will have more powerful effects than delivery of either alone.

The network showed that fears of a prolonged death (item 12) tend to be associated with concerns about how this would burden others (item 8) and its negative impact on loved ones (item 9). This result is consistent with earlier studies documenting an association between worries about the future and the experience or anticipation of stress imposed on others, which relates to perceived burdensomeness $[33,34]$. Another important set of linkages emerged between the fear of dying alone (item 13), having not said important things to others (item 2), and regret about things that were not done (item 1). This suggests that a sense of unfinished business for individuals may often involve unresolved or problematic relationships. Interventions that aim to support patients in achieving a sense of social connectedness and life completion may require understanding how individuals view their social legacy and the possibilities for reconnection or letting go of the things that cannot be changed in life $[12,13,35,36]$. Discussion of the practical issues of end-of-life preparation may contribute to interventions that focus to help patients in defining what is important to them in the time that is left [3].

The presence of a single central node is a unique feature of the present network [37, 38]. The experience of time running out may be central to the psychology of incurable disease, which may fundamentally be a problem of time [39]. Nonetheless, patients with advanced cancer may present 
with different individual configurations or profiles of death-related concerns. For some, a foreshortened future can disrupt the sense of meaning attached to previously held values and goals in life, causing existential regret $[19,39,40]$. Others may be more overwhelmed by the uncertainty and losses that lie ahead, including concerns about how others will respond to unfolding events[18], as suggested by the links between being concerned about "my own death and dying", "not knowing about what happens at the end of life", and the "impact on loved ones" in the network.

We note the following limitations and future research directions. The present network shows how concerns are linked on average in the sample, although a range of individual death anxiety profiles may be expected. This overview of conceptual associations between concerns may provide a fruitful departure for further investigations that focus on how individuals may differ in their patterns of death anxiety. Network structures of death-related concerns may further be compared across subgroups with high vs. low physical symptom burden, time since diagnosis, and desire for hastened death. Further, time series will enable insight into single-person networks. In addition, networks including a broader range of psychological and existential distress symptoms could further explore the structure of distress in advanced cancer. We are aware that the 15-item-DADDS may not cover all dimensions of death anxiety in advanced cancer. However, its shortness and conceptual parsimony avoid overlap with other distress concepts. While this applies to all studies using self-report measures, semantic overlap between items is a source of correlation that must be taken into account in interpretation, in addition to the association among concepts. Lastly, our results are most representative of outpatients with advanced cancer with an expected survival of 12-18 months interested in receiving psychotherapeutic intervention. The structure of concerns may change with disease progression and differ in patients closer to death.

In conclusion, our study offers an overview of the richness and complexity of associations involved in the structure of death-related distress in individuals with advanced cancer by a detailed investigation of the links between specific fears and worries. The map of concerns and their connectivity that has 
been generated, may help to guide clinical conversations and the development of supportive interventions to alleviate these symptoms.

\section{Acknowledgements}

We would like to thank the participants for their time and effort to complete this study.

\section{Conflicts of interest}

The authors declare no conflict of interest.

\section{Funding}

This study was funded by research grants from the Canadian Institutes of Health Research (CIHR \#MOP 106473) and the German Research Society (DFG). This research was also supported in part by the Princess Margaret Cancer Foundation (Al Hertz, Kirchmann and Kirsh Family Funds), the Campbell Family Cancer Research Institute and the Ontario Cancer Institute at the Princess Margaret Cancer Centre, and by the Ontario Ministry of Health and Long Term Care (OMOHLTC). The views expressed do not necessarily reflect those of the OMOHLTC. Dr. Rodin is also supported by the University of Toronto/UHN Harold and Shirley Lederman Chair in Psychosocial Oncology and Palliative Care. The funders had no role in the final design, analysis, and writing of this study. The views expressed do not necessarily reflect those of the funders.

\section{Contributors}

Conception and design: SV, GR, CL. Data analysis: SV, CL. Interpretation and manuscript writing: SV, CM, JS, SW, TP, AC, AR, CZ, SH, GR, CL. Final approval: SV, CM, JS, SW, TP, AC, AR, CZ, SH, GR, CL. Guarantor: SV, GR, CL.

\section{Ethics approval}


University Health Network Research Ethics Board. 


\section{References}

1 Bernacki RE, Block SD. Communication about serious illness care goals: a review and synthesis of best practices. JAMA Intern Med 2014;174(12):1994-2003.

2 Rodin G, Zimmermann C. Psychoanalytic reflections on mortality: a reconsideration. J Am Acad Psychoanal Dyn Psychiatry 2008;36(1):181-96.

3 Nissim R, Freeman E, Lo C, et al. Managing Cancer and Living Meaningfully (CALM): a qualitative study of a brief individual psychotherapy for individuals with advanced cancer. Palliat Med 2012;26(5):713-21.

4 Henoch I, Danielson E. Existential concerns among patients with cancer and interventions to meet them: an integrative literature review. Psychooncology 2009;18(3):225-36. doi:10.1002/pon.1424.

5 Gawande A. Being mortal: Medicine and what matters in the end, 1st edn. New York, NY: Metropolitan Books 2014.

6 Iverach L, Menzies RG, Menzies RE. Death anxiety and its role in psychopathology: reviewing the status of a transdiagnostic construct. Clin Psychol Rev 2014;34(7):580-93.

7 Copp G. A review of current theories of death and dying. J Adv Nurs 1998;28(2):382-90.

8 Fakhri S, Engelberg RA, Downey L, et al. Factors Affecting Patients' Preferences for and Actual Discussions About End-of-Life Care. J Pain Symptom Manage 2016:10.1016/j.jpainsymman.2016.03.012.

9 Shaw C, Chrysikou V, Davis S, et al. Inviting end-of-life talk in initial CALM therapy sessions: a conversation analytic study. Patient Educ Couns 2017;100(2):259-266.

10 Classen CC, Kraemer HC, Blasey C, et al. Supportive-expressive group therapy for primary breast cancer patients: a randomized prospective multicenter trial. Psychooncology 2008;17(5):438-47.

11 Kissane DW, Bloch S, Smith GC, et al. Cognitive-existential group psychotherapy for women with primary breast cancer: a randomised controlled trial. Psychooncology 2003;12(6):532-46. 
12 Breitbart W, Rosenfeld B, Pessin H, et al. Meaning-Centered Group Psychotherapy: an effective intervention for improving psychological well-being in patients with advanced cancer. J Clin Oncol 2015;33(7):749-54.

13 Lo C, Hales S, Chiu A, et al. Managing Cancer And Living Meaningfully (CALM): randomised feasibility trial in patients with advanced cancer. BMJ Supp Pall Care 2016;doi:10.1136/bmjspcare-2015-000866.

14 Lo C, Hales S, Zimmermann C, et al. Measuring death-related anxiety in advanced cancer: preliminary psychometrics of the Death and Dying Distress Scale. Journal of Pediatric Hematology/Oncology 2011;33:140-45.

15 Krause S, Rydall A, Hales S, et al. Initial validation of the Death and Dying Distress Scale for the assessment of death anxiety in patients with advanced cancer. J Pain Symptom Manage 2015;49(1):126-34.

16 Garcia-Rueda N, Carvajal Valcarcel A, Saracibar-Razquin M, et al. The experience of living with advanced-stage cancer: a thematic synthesis of the literature. Eur J Cancer Care 2016;25(4):551-69.

17 Moestrup L, Hansen HP. Existential concerns about death: a qualitative study of dying patients in a Danish hospice. Am J Hosp Palliat Care 2015;32(4):427-36.

18 Tong E, Deckert A, Gani N, et al. The meaning of self-reported death anxiety in advanced cancer. Palliat Med 2016;30(8):772-79.

19 Blinderman CD, Cherny NI. Existential issues do not necessarily result in existential suffering: lessons from cancer patients in Israel. Palliat Med 2005;19(5):371-80.

20 Nissim R, Rennie D, Fleming S, et al. Goals set in the land of the living/dying: a longitudinal study of patients living with advanced cancer. Death Stud 2012;36(4):360-90.

21 Robinson S, Kissane DW, Brooker J, et al. A review of the construct of demoralization: history, definitions, and future directions for palliative care. Am J Hosp Palliat Care 2016;33(1):93-101.

22 Chochinov HM, McClement SE, Hack TF, et al. The Patient Dignity Inventory: applications in the oncology setting. J Palliat Med 2012;15(9):998-1005. 
23 Lo C, Burman D, Swami N, et al. Validation of the QUAL-EC for assessing quality of life in patients with advanced cancer. Eur J Cancer 2011;47(4):554-60.

24 Boston P, Bruce A, Schreiber R. Existential suffering in the palliative care setting: an integrated literature review. J Pain Symptom Manage 2011;41(3):604-18.

25 Lo C, Hales S, Rydall A, et al. Managing Cancer And Living Meaningfully: study protocol for a randomized controlled trial. Trials 2015;16(1):391.

26 R Development Core Team. R: A language and environment for statistical computing. Vienna, Austria 2008.

27 Epskamp S, Cramer AOJ, Waldorp LJ, et al. qgraph: network visualizations of relationships in psychometric data. J Stat Soft 2012;48(4):1-18.

28 Borsboom D, Cramer AOJ. Network analysis: an integrative approach to the structure of psychopathology. Annu Rev Clin Psychol 2013;9:91-121.

29 Chang VT, Hwang SS, Feuerman M, et al. The memorial symptom assessment scale short form (MSAS-SF). Cancer 2000;89(5):1162-71.

30 Lee V, Loiselle CG. The salience of existential concerns across the cancer control continuum. Palliat Support Care 2012;10(2):123-33.

31 Hales S. Attachment and the End of Life Experience. In: Hunter J, Maunder R, eds. Improving Patient Treatment with Attachment Theory, 1st edn. Cham: Springer International Publishing 2016:93-103.

32 Zimmermann C, Swami N, Krzyzanowska M, et al. Early palliative care for patients with advanced cancer: a cluster-randomised controlled trial. The Lancet 2014;383(9930):1721-30.

33 McPherson CJ, Wilson KG, Murray MA. Feeling like a burden: exploring the perspectives of patients at the end of life. Soc Sci Med 2007;64(2):417-27.

34 Tang S, Chen J, Chou W, et al. Longitudinal analysis of severe anxiety symptoms in the last year of life among patients with advanced cancer: relationships with proximity to death, burden, and social support. J Natl Compr Canc Netw 2016;14(6):727-34. 
35 Keall RM, Clayton JM, Butow PN. Therapeutic life review in palliative care: a systematic review of quantitative evaluations. J Pain Symptom Manage 2015;49(4):747-61.

36 Chochinov HM, Kristjanson LJ, Breitbart W, et al. Effect of dignity therapy on distress and endof-life experience in terminally ill patients: a randomised controlled trial. Lancet Oncol 2011;12(8):753-62.

37 Fried EI, Epskamp S, Nesse RM, et al. What are 'good' depression symptoms? Comparing the centrality of DSM and non-DSM symptoms of depression in a network analysis. $J$ Affect Disord 2016;189:314-20.

38 Heisig SR, Shedden-Mora MC, Blanckenburg P von, et al. What do women with breast cancer expect from their treatment? Correlates of negative treatment expectations about endocrine therapy. Psychooncology 2016:doi:10.1002/pon.4089.

39 Willig C. "My bus is here": a phenomenological exploration of "living-with-dying". Health Psychol 2015;34(4):417-25.

40 Kissane DW. The relief of existential suffering. Arch Intern Med 2012;172(19):1501-05. 


\section{Figure Captions}

Figure 1: Frequency of death-related concerns $(\mathrm{N}=382)$. Moderate distress is defined by item ratings $\geq 3$. The question stem for items 1 to 10 was: "Over the past two weeks, how distressed did you feel about...". The question stem for items 11 to 15 was: "Over the past two weeks, how distressed did you feel that your own death and dying may...".

Figure 2: Network of death-related concerns. Nodes represent the death-related items; edges represent partial correlations between items. Thicker edges mean higher partial correlations. The question stem for items 1 to 10 (white) was: "Over the past two weeks, how distressed did you feel about...". The question stem for items 11 to 15 (grey) was: "Over the past two weeks, how distressed did you feel that your own death and dying may...". Partial correlations $<0.05$ are not shown. All edge weights are listed in the online supplement. See Figure 1 for complete items.

Figure 3: Closeness-centrality and clustering coefficients for death-related concerns. Closenesscentrality indicates a node's connectivity within the network. It describes the node's total distance to all other nodes in the network. Higher values indicate greater centrality. The clustering coefficient indicates a node's redundancy relative to other nodes. It describes the percentage of nonzero connections that exist between its neighbor nodes. Higher values indicate greater clustering. 
9. The impact of my death on my loved ones

12. Be prolonged or drawn out 14. Happen with a lot of pain or suffering 8. Being a burden to others 10. My own death and dying 5. Not having a future 7. Running out of time 4. Not knowing what happens near the end of life

1. Not having done all the things I wanted to do 6. The missed opportunities in my life 3. Not having achieved my life goals and ambitions 15. Happen very soon 2. Not having said all I wanted to say to the people I care about 11. Happen suddenly or unexpectedly 13. Happen when I am alone

$55.3 \%$ $54.8 \%$

$51.2 \%$ $49.6 \%$ $49.1 \%$ $47.8 \%$ $46.4 \%$ $45.1 \%$ $40.9 \%$

$36.7 \%$

$34.3 \%$

$27.2 \%$

$25.3 \%$

$22.4 \%$ 


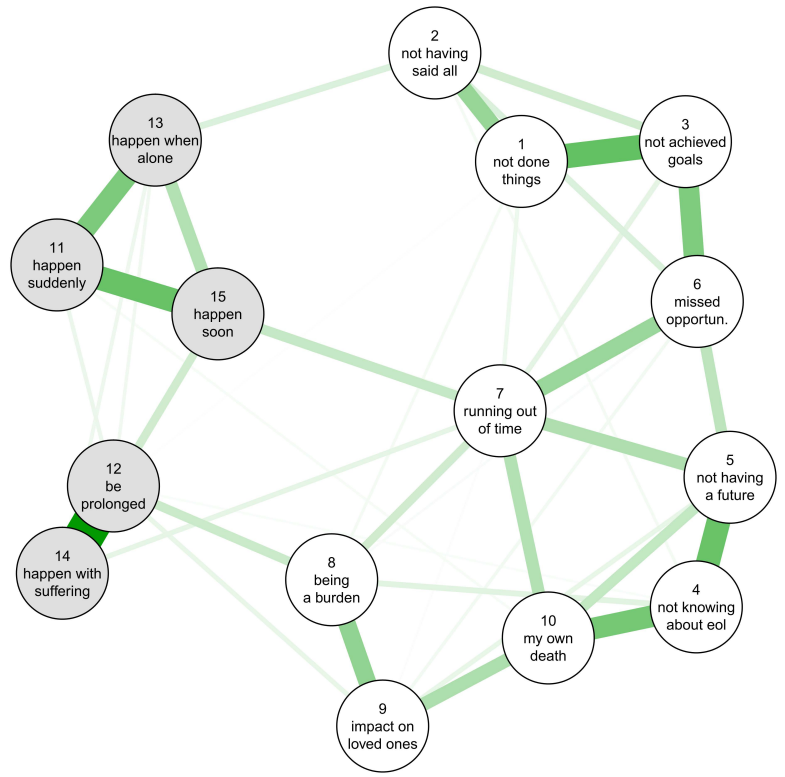




\section{Closeness-Centrality}

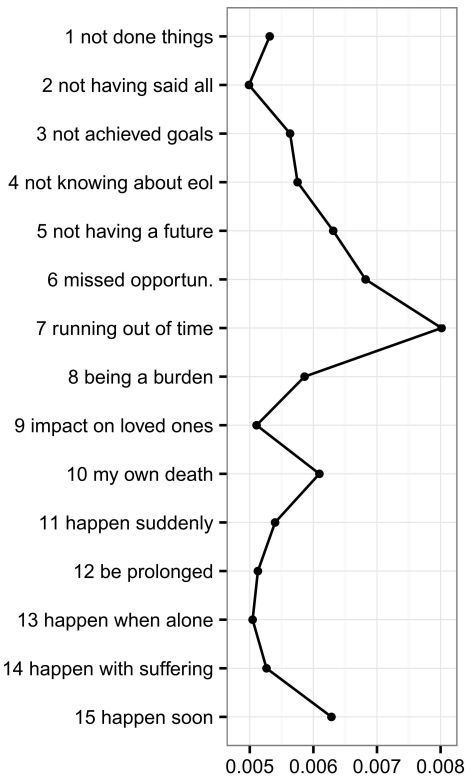

Clustering

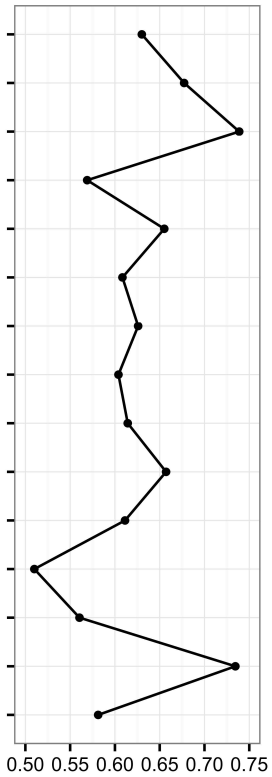




\section{Online supplemental material for Vehling et al.: "A concept map of death-related anxieties"}

Table 2: Descriptive statistics for death-related concerns

\begin{tabular}{lrrrr}
\hline Death-related concerns & Mean & SD & Skew & Kurtosis \\
\hline 9. The impact of my death on my loved ones. & 3.1 & 1.7 & -0.5 & -1.0 \\
14. Happen with a lot of pain or suffering. & 2.5 & 1.8 & -0.1 & -1.4 \\
8. Being a burden to others. & 2.4 & 1.7 & -0.0 & -1.2 \\
10. My own death and dying. & 2.4 & 1.7 & 0.0 & -1.2 \\
12. Be prolonged or drawn out. & 2.4 & 1.7 & -0.1 & -1.2 \\
5. Not having a future. & 2.3 & 1.7 & 0.0 & -1.3 \\
7. Running out of time. & 2.3 & 1.7 & 0.7 & -1.2 \\
1. Not having done all the things I wanted to do. & 2.2 & 1.5 & 0.1 & -0.9 \\
4. Not knowing what happens near the end of life. & 2.2 & 1.7 & 0.1 & -1.2 \\
6. The missed opportunities in my life. & 2.0 & 1.7 & 0.3 & -1.2 \\
3. Not having achieved my life goals and ambitions. & 1.9 & 1.6 & 0.4 & -1.0 \\
15. Happen very soon. & 1.8 & 1.7 & 0.5 & -1.0 \\
2. Not having said all I wanted to say to the people I care about. & 1.6 & 1.4 & 0.6 & -0.6 \\
11. Happen suddenly or unexpectedly. & 1.4 & 1.5 & 0.8 & -0.4 \\
13. Happen when I am alone. & 1.2 & 1.5 & 1.1 & -0.1 \\
Total mean score of death anxiety & 31.6 & 18.0 & 0.2 & -0.8 \\
Number of death-related concerns ${ }^{a}$ & 6.5 & 4.7 & 0.1 & -1.3 \\
\hline
\end{tabular}

${ }^{\mathrm{a}}$ Number of concerns rated $\geq 3$, indicating at least moderate distress 
Table 3: Correlations among death-related concerns

\begin{tabular}{|c|c|c|c|c|c|c|c|c|c|c|c|c|c|c|}
\hline Item & 2 & 3 & 4 & 5 & 6 & 7 & 8 & 9 & 10 & 11 & 12 & 13 & 14 & 15 \\
\hline 1 & .55 & .65 & .44 & .52 & .54 & .56 & .47 & .41 & .41 & .37 & .42 & .29 & .39 & .41 \\
\hline 2 & - & .54 & .45 & .45 & .52 & .48 & .42 & .37 & .42 & .37 & .32 & .41 & .29 & .37 \\
\hline 3 & & - & .47 & .57 & .67 & .62 & .47 & .42 & .45 & .38 & .32 & .40 & .35 & .41 \\
\hline 4 & & & - & .73 & .57 & .63 & .56 & .58 & .71 & .45 & .50 & .42 & .42 & .44 \\
\hline 5 & & & & - & .68 & .73 & .55 & .62 & .70 & .48 & .47 & .43 & .44 & .55 \\
\hline 6 & & & & & - & .71 & .55 & .56 & .58 & .44 & .39 & .43 & .37 & .49 \\
\hline 7 & & & & & & - & .62 & .62 & .69 & .48 & .53 & .46 & .55 & .60 \\
\hline 8 & & & & & & & - & .62 & .47 & .38 & .55 & .37 & .50 & .44 \\
\hline 9 & & & & & & & & - & .63 & .41 & .53 & .36 & .50 & .49 \\
\hline 10 & & & & & & & & & - & .48 & .46 & .45 & .42 & .52 \\
\hline 11 & & & & & & & & & & - & .49 & .60 & .40 & .65 \\
\hline 12 & & & & & & & & & & & - & .49 & .74 & .52 \\
\hline 13 & & & & & & & & & & & & - & .49 & .58 \\
\hline 14 & & & & & & & & & & & & & - & .55 \\
\hline
\end{tabular}

Note: All correlations were significant $(\mathrm{p}<.001)$.

1. Not having done all the things I wanted to do

2. Not having said all I wanted to say to the people I care about

3. Not having achieved my life goals and ambitions

4. Not knowing what happens near the end of life

5. Not having a future

6. The missed opportunities in my life

7. Running out of time

8. Being a burden to others

9. The impact of my death on my loved ones

10. My own death and dying

11. Happen suddenly or unexpectedly

12. Be prolonged or drawn out

13. Happen when I am alone

14. Happen with a lot of pain or suffering

15. Happen very soon 
Table 4: Regularized partial correlations among death-related concerns (edge weights of the network shown in Figure 1)

\begin{tabular}{|c|c|c|c|c|c|c|c|c|c|c|c|c|c|c|}
\hline Item & 2 & 3 & 4 & 5 & 6 & 7 & 8 & 9 & 10 & 11 & 12 & 13 & 14 & 15 \\
\hline 1 & .23 & .32 & 0 & .05 & .01 & .08 & .07 & 0 & 0 & 0 & .06 & 0 & 0 & .01 \\
\hline 2 & - & .14 & .07 & 0 & .11 & 0 & .03 & 0 & 0 & .02 & 0 & .11 & 0 & 0 \\
\hline 3 & & - & 0 & .05 & .28 & .10 & .01 & 0 & 0 & 0 & -.02 & .03 & 0 & 0 \\
\hline 4 & & & - & .32 & .02 & 0 & .10 & .04 & .29 & .03 & .06 & 0 & 0 & 0 \\
\hline 5 & & & & - & .17 & .19 & 0 & .09 & .16 & .02 & 0 & 0 & 0 & .06 \\
\hline 6 & & & & & - & .23 & .06 & .07 & .01 & .01 & 0 & .01 & 0 & 0 \\
\hline 7 & & & & & & - & .13 & .06 & .18 & 0 & 0 & 0 & .10 & .15 \\
\hline 8 & & & & & & & - & .24 & 0 & 0 & .14 & 0 & .03 & 0 \\
\hline 9 & & & & & & & & - & .19 & 0 & .09 & 0 & .04 & .03 \\
\hline 10 & & & & & & & & & - & .06 & 0 & .03 & 0 & .02 \\
\hline 11 & & & & & & & & & & - & .08 & .28 & 0 & .32 \\
\hline 12 & & & & & & & & & & & - & .07 & .50 & .02 \\
\hline 13 & & & & & & & & & & & & - & .08 & .18 \\
\hline 14 & & & & & & & & & & & & & - & .13 \\
\hline
\end{tabular}

Note: Greater edge weights result in thicker edges in the network shown in Figure 1.

1. Not having done all the things I wanted to do

2. Not having said all I wanted to say to the people I care about

3. Not having achieved my life goals and ambitions

4. Not knowing what happens near the end of life

5. Not having a future

6. The missed opportunities in my life

7. Running out of time

8. Being a burden to others

9. The impact of my death on my loved ones

10. My own death and dying

11. Happen suddenly or unexpectedly

12. Be prolonged or drawn out

13. Happen when I am alone

14. Happen with a lot of pain or suffering

15. Happen very soon 